\title{
Genome-wide association and genomic prediction of resistance to viral nervous necrosis in European sea bass (Dicentrarchus labrax) using RAD sequencing
}

Christos Palaiokostas $^{1 *}$ (D), Sophie Cariou ${ }^{2}$, Anastasia Bestin ${ }^{3}$, Jean-Sebastien Bruant ${ }^{2}$, Pierrick Haffray ${ }^{3}$, Thierry Morin ${ }^{4}$, Joëlle Cabon ${ }^{4}$, François Allal ${ }^{5}$, Marc Vandeputte ${ }^{6}$ and Ross D. Houston ${ }^{1}$

\begin{abstract}
Background: European sea bass (Dicentrarchus labrax) is one of the most important species for European aquaculture. Viral nervous necrosis (VNN), commonly caused by the redspotted grouper nervous necrosis virus (RGNNV), can result in high levels of morbidity and mortality, mainly during the larval and juvenile stages of cultured sea bass. In the absence of efficient therapeutic treatments, selective breeding for host resistance offers a promising strategy to control this disease. Our study aimed at investigating genetic resistance to VNN and genomic-based approaches to improve disease resistance by selective breeding. A population of 1538 sea bass juveniles from a factorial cross between 48 sires and 17 dams was challenged with RGNNV with mortalities and survivors being recorded and sampled for genotyping by the RAD sequencing approach.
\end{abstract}

Results: We used genome-wide genotype data from 9195 single nucleotide polymorphisms (SNPs) for downstream analysis. Estimates of heritability of survival on the underlying scale for the pedigree and genomic relationship matrices were 0.27 (HPD interval 95\%: 0.14-0.40) and 0.43 (0.29-0.57), respectively. Classical genome-wide association analysis detected genome-wide significant quantitative trait loci (QTL) for resistance to VNN on chromosomes (unassigned scaffolds in the case of 'chromosome' 25) 3,20 and $25(P<1 \mathrm{e} 06)$. Weighted genomic best linear unbiased predictor provided additional support for the QTL on chromosome 3 and suggested that it explained $4 \%$ of the additive genetic variation. Genomic prediction approaches were tested to investigate the potential of using genome-wide SNP data to estimate breeding values for resistance to VNN and showed that genomic prediction resulted in a 13\% increase in successful classification of resistant and susceptible animals compared to pedigree-based methods, with Bayes A and Bayes B giving the highest predictive ability.

Conclusions: Genome-wide significant QTL were identified but each with relatively small effects on the trait. Tests of genomic prediction suggested that incorporating genome-wide SNP data is likely to result in higher accuracy of estimated breeding values for resistance to VNN. RAD sequencing is an effective method for generating such genomewide SNPs, and our findings highlight the potential of genomic selection to breed farmed European sea bass with improved resistance to VNN.

\footnotetext{
*Correspondence: christos.palaiokostas@slu.se

${ }^{1}$ The Roslin Institute, Royal (Dick) School of Veterinary Studies, University

of Edinburgh, Easter Bush, Midlothian EH25 9RG, Scotland, UK

Full list of author information is available at the end of the article
} 


\section{Background}

European sea bass (Dicentrarchus labrax) is a popular and valuable species for Mediterranean aquaculture, with a production volume of more than 150,000 tons [1]. Viral nervous necrosis (VNN) is a commonlyencountered pathogen, which has been detected in more than 70 wild or cultured marine and fresh water species [2]. Frequent mass mortalities of sea bass due to VNN have been reported, especially during the summer period, with fish being particularly susceptible during the larval and juvenile stages [3]. The disease is considered to be a primary problem in Mediterranean mariculture [4] and, to date, there is no fully effective therapeutic agent or vaccine to tackle it [5]. Selective breeding can be a valuable tool to prevent the detrimental effects of disease outbreaks in farmed livestock and fish [6]. Moderate to high heritabilities for disease resistance have been reported in numerous aquaculture species, which indicates that genetic progress is possible through selective breeding [7]. For resistance to VNN in sea bass, a moderate heritability of 0.26 was recently reported [8], which implies that selective breeding has potential as a component of $\mathrm{VNN}$ prevention and control.

Recent technological advances in genome-wide sequencing and genotyping technology have facilitated cost-effective generation of genome-wide marker data, even in non-model organisms [9]. Such genotyping data can be used in selective breeding programs to increase the accuracy of breeding value predictions for selection candidates, as compared to the classical breeding approach where breeding values are typically estimated at the family level using pedigree data $[10,11]$. The genetic architecture of most traits relevant to farmed animal production (e.g. growth and disease resistance) is polygenic $[12,13]$, which explains why the application of markerassisted selection in aquaculture has had limited success, with a few exceptions associated with major-effect loci [14-17]. Therefore, as for terrestrial livestock, genomic prediction [18] is an effective approach to improve selection accuracy compared to traditional pedigree-based approaches in aquaculture breeding programs [19-21].

Restriction-site associated DNA (RAD) sequencing is a reduced representation high-throughput sequencing technique for the concurrent detection and genotyping of single nucleotide polymorphisms (SNPs) in multiplexed samples, each containing a unique nucleotide barcode [22]. RAD sequencing and similar genotyping-by-sequencing techniques rely on the digestion of genomic DNA with a restriction enzyme and subsequent high-depth sequencing of the flanking regions. Such genotyping-by-sequencing techniques have been applied in a wide range of aquaculture species [23], both for genome-wide association studies (GWAS) [24-26] and genomic selection studies [27-31].

The aim of this study was to investigate genetic resistance to $\mathrm{VNN}$ in sea bass juveniles, using a RAD sequencing approach to generate genome-wide SNP data from 1538 individual disease-challenged fish. Heritability estimates were obtained using both pedigree and genomic relationship matrices for survival status (dead/alive) on the underlying liability scale. GWA approaches were used to test associations of both individual SNPs and genomic regions with resistance to VNN. Finally, genomic prediction of breeding values for resistance to VNN was tested using several approaches to evaluate the potential of genomic selection for genetic improvement of resistance to VNN in seabass.

\section{Methods}

\section{Sample collection and disease challenge}

The population of fish for the VNN disease challenge was obtained by artificial fertilization of 18 dams and 49 sires from the Ferme Marine de Douhet breeding nucleus (Oléron, France). Five factorial mating blocks, each comprising 9 to 10 sires and 3 to 5 dams, were created in February 2014. The fertilized eggs of each dam were transferred to individual incubators. Following egg hatching, equal numbers of larvae from each incubator were transferred in a single tank and reared in common environmental conditions.

The fish (at a size of $\sim 10 \mathrm{~g}$ ) were challenged at the Unité de Pathologies Virales des Poissons-ANSES facility (Plouzané, France). Autopsies and extensive bacteriological and virological analyses were carried out on receipt of fish to confirm their health status. After 4 weeks of acclimatization, 1990 individuals distributed in three tanks were immersed for 2 to $3 \mathrm{~h}$ in static seawater, containing $1 \times 10^{5} 50 \%$ tissue culture infective dose $\left(\mathrm{TCID}_{50}\right)$ per $\mathrm{ml}$ of the W80 betanodavirus strain, which was produced and titered in striped snakehead cells (SSN-1), as previously described [32]. Strain W80 belongs to the redspotted grouper nervous necrosis virus (RGNNV) genotype, the most common NNV type in the Mediterranean area [33]. A negative control tank that consisted of sea bass juveniles from the same population $(n=200)$ immersed with non-infected SSN-1-cell supernatant was also included. After infection, all fish were maintained at $27 \pm 1{ }^{\circ} \mathrm{C}$ in an open water circuit. A pre-test of the experimental conditions was conducted to confirm the virulence of the pathogen.

\section{RAD library preparation and sequencing}

DNA was extracted from fin samples of the challenged fish using the REALPure genomic DNA extraction kit (Durviz S.L.) and treated with RNase. Each sample was 
quantified by spectrophotometry (Nanodrop), quality-assessed by agarose gel electrophoresis, and finally diluted to a concentration of $20 \mathrm{ng} / \mu \mathrm{L}$ in $5 \mathrm{mmol} / \mathrm{L}$ Tris, pH 8.5 using a Qubit Fluorometer (Invitrogen).

The protocol for RAD library preparation followed the methodology originally described in Baird et al. [22]. Briefly, each sample $(0.72 \mu \mathrm{g}$ parental DNA per $0.24 \mu \mathrm{g}$ offspring DNA) was digested at $37{ }^{\circ} \mathrm{C}$ for 60 min with the high-fidelity restriction enzyme Sbfl (that recognises the CCTGCA|GG motif) - (New England Biolabs; NEB) using 6 U Sbf I per $\mu$ g genomic DNA in $1 \times$ Reaction Buffer 4 (NEB) at a final concentration of $1 \mu \mathrm{g}$ DNA per $50 \mu \mathrm{L}$ reaction volume. Reactions $(12 \mu \mathrm{L}$ final volumes) were then heat-inactivated at $65{ }^{\circ} \mathrm{C}$ for $20 \mathrm{~min}$. Individual specific P1 adapters, each with a unique 5-7 bp barcode, were ligated to the $S b f$ I-digested DNA at $20{ }^{\circ} \mathrm{C}$ for $60 \mathrm{~min}$ by adding $1.8 / 0.6 \mu \mathrm{L}$ of $100 \mathrm{nmol} / \mathrm{L}$ P1 adapter, $0.45 / 0.15 \mu \mathrm{L}$ of $100 \mathrm{mmol} / \mathrm{L} \mathrm{rATP}$ (Promega), $0.75 / 0.25 \mu \mathrm{L} 10 \times$ reaction buffer 2 (NEB), 0.36/0.12 $\mu \mathrm{L}$ T4 ligase (NEB, $2 \mathrm{M} \mathrm{U} / \mathrm{mL}$ ) and reaction volumes for each parental/offspring sample were completed to $45 / 15$ $\mu \mathrm{L}$ with nuclease-free water. Following heat inactivation at $65{ }^{\circ} \mathrm{C}$ for $20 \mathrm{~min}$, ligation reactions were slowly cooled down to room temperature (over $1 \mathrm{~h}$ ), then combined in appropriate multiplex pools. Shearing (Covaris S2 sonication) and initial size selection (300 to $600 \mathrm{bp}$ ) by agarose gel separation was followed by gel purification, end repair, dA overhang addition, P2 (individual specific adapters) paired-end adapter ligation, library amplification, as in the original RAD protocol [22, 34]. A volume of $150 \mu \mathrm{L}$ of each amplified library (16 to 18 PCR cycles, library-dependent) was size-selected (400 to $700 \mathrm{bp}$ ) by gel electrophoresis [35]. Following a final gel elution step into $20 \mu \mathrm{L}$ EB buffer (MinElute Gel Purification Kit, Qiagen), 66 libraries (24 animals each) were sent to BMR in Italy, for sequencing. Libraries were run in 14 lanes of an Illumina NextSeq 500, using 75 base paired-end reads (v2 chemistry). The sequence reads were deposited at the NCBI Sequence Read Archive (SRA) under the accession number PRJNA407892.

\section{SNP identification and genotyping}

Sequence reads of low quality, with a missing restriction site, or with ambiguous barcodes and PCR duplicates were discarded. The remaining reads were aligned to the European sea bass reference genome [36] assembly GCA_000689215.1 using the Bowtie2 program [37]. Aligned reads were sorted into RAD loci and genotypes were called using Stacks software 1.4 [38]. The likelihoodbased SNP calling algorithm [39] implemented in Stacks evaluates each nucleotide position at each RAD-locus of all individuals, using a maximum likelihood approach to differentiate true SNPs from putative sequencing errors. SNP data for downstream analyses were obtained after the following quality control (QC) steps: RAD loci were formed using a minimum stack depth of 10 (parental samples) or 5 (offspring samples) reads. Only RAD loci with a maximum of two SNPs were considered for downstream analysis. SNPs that had a minor allele frequency (MAF) less than 0.05 and more than $25 \%$ missing data and those that deviated from expected Hardy-Weinberg equilibrium $(P<1$ e- 06 ; parental samples) were discarded.

\section{Parentage assignment}

Parentage assignment was performed with the $\mathrm{R} / \mathrm{h}$ sphase program [40] using all SNPs that passed QC and allowing for a maximum genotyping error of $3.5 \%$. The pedigree obtained using this approach was further validated for potential erroneous assignments using the FImpute software [41].

\section{Estimation of heritabilities}

Variance components were estimated using the R/BGLR software [42]. The probit link function was used to connect the observed binary phenotype $(0=$ dead, $1=$ alive $)$ with the latent variable (the underlying liability). The following model was applied:

$$
\mathbf{l}=\mathbf{X b}+\mathbf{Z u}+\mathbf{e},
$$

where $\mathbf{l}$ is a vector of latent variables, $\mathbf{b}$ a vector of the fixed effects (intercept and tank), $\mathbf{X}$ the incidence matrix relating phenotypes with the fixed effects, $\mathbf{Z}$ the incidence matrix relating phenotypes with the random animal genetic effects, $\mathbf{u}$ the vector of random animal genetic effects with the following distribution $N\left(0, \mathbf{A} \sigma_{\mathrm{g}}^{2}\right)$, where $\mathbf{A}$ is the pedigree-based relationship matrix, which was replaced with the genomic relationship matrix G [43] for certain analyses as described below, $\sigma_{\mathrm{g}}^{2}$ is the additive genetic variance, and $\mathbf{e}$ is a vector of residuals with the following distribution $N\left(0, \mathbf{I}_{\mathrm{e}}^{2}\right)$, where $\sigma_{\mathrm{e}}^{2}$ is the residual variance and $\mathbf{I}$ the identity matrix.

The parameters of this model were estimated by Monte Carlo Markov chain (MCMC) Gibbs sampling (11 million iterations; burn-in: 1 million; thin: 1000). Convergence of the resulting posterior distributions was assessed both visually (inspecting the resulting MCMC plots) and analytically using the R/coda v0.19-1 software [44]. Heritability for survival on the underlying scale was estimated as:

$$
h^{2}=\frac{\sigma_{\mathrm{g}}^{2}}{\sigma_{\mathrm{g}}^{2}+\sigma_{\mathrm{e}}^{2}},
$$


where $\sigma_{\mathrm{g}}^{2}$ is the estimate of the additive genetic variance and $\sigma_{\mathrm{e}}^{2}$ the residual variance, which was set equal to 1 because it is not identifiable in threshold models $[45,46]$.

\section{Genome-wide association study (GWAS)}

To test the association of individual SNPs with resistance to VNN, a 'classical' genome-wide association study (CGWAS) was performed using the R/gaston software [47]. The mixed model applied for overall survival on the observed scale had the same format as in Eq. (1) but with the addition of the genotype of an individual SNP as a fixed effect. Variance components were estimated using the penalized quasi-likelihood approach [48]. The genome-wide significance threshold was calculated using a Bonferroni correction $(0.05 / \mathrm{N})$, where $\mathrm{N}$ represents the number of QC-filtered SNPs across the genome.

In addition to the CGWAS approach, weighted genomic best linear unbiased predictor (WGBLUP) was performed [49] to estimate SNP effects by using genomic estimated breeding values (GEBV) [50]. SNP weights were estimated using non-overlapping windows of 10 adjacent SNPs. Explained additive genetic variance was estimated using subsequent non-overlapping windows including adjacent SNPs within a distance of $0.5 \mathrm{Mb}$. Initially, the weighted genomic relationship matrix was created following the method of VanRaden [43] as:

$$
\mathbf{G}^{*}=\mathbf{Z D Z}^{\prime} \mathbf{q}
$$

where $\mathbf{Z}$ is the design matrix relating genotypes of each SNP with phenotypes, $\mathbf{D}$ is a weight matrix for all SNPs, and $\mathbf{q}$ is a weighting vector derived from the observed SNP frequencies. Briefly, WGBLUP was carried out as follows [49]:

a. Initialize $\mathbf{D}=\mathbf{I}$ and $\mathbf{t}=1$, where $\mathbf{I}$ is the identity matrix and $t$ is the iteration number;

b. Calculate $\mathbf{G}^{*}$;

c. Estimate GEBV using GBLUP;

d. Estimate the SNP effects from GEBV based on the equation $\hat{\boldsymbol{\alpha}}=\mathbf{q D Z} \mathbf{G}^{*} \hat{\mathbf{u}}$, where $\hat{\boldsymbol{\alpha}}$ represents the vector of SNP effects and $\hat{\mathbf{u}}$ vector of GEBV;

e. Calculate the weight for matrix $\mathbf{D}$ for each SNP: $d_{i i}^{(t+1)}=\frac{\sum_{i=1}^{n} u_{i}^{2}}{n}$, where $n=10$ represents number of adjacent SNPs;

f. Normalize the weights of SNPs such that the total genetic variance remains constant;

g. Loop to step (d) (three iterations).

The percentage of additive genetic variance explained by each genomic region was estimated by non-overlapping windows of $0.5 \mathrm{Mb}$ as follows:

$$
\frac{\operatorname{Var}\left(\alpha_{\mathrm{i}}\right)}{\sigma_{\alpha}^{2}} \times 100 \%=\frac{\operatorname{Var}\left(\sum_{\mathrm{i}}^{\mathrm{i}=\mathrm{w}} \mathrm{z}_{\mathrm{i}} \widehat{\mathrm{u}_{\mathrm{i}}}\right)}{\sigma_{\alpha}^{2}} \times 100 \%,
$$

where $\mathrm{w}$ denotes group of SNPs within the tested window.

This analysis was performed using the THRGIBBS1F90 program from the BLUPF90 software suite $[51,52]$ to estimate the GEBV, combined with three iterations using PreGSF90 and PostGSF90 [53].

\section{Genomic prediction}

To assess the potential of genomic selection for improved resistance to $\mathrm{VNN}$ in sea bass breeding programs, the accuracy of GEBV was assessed and compared to EBV obtained with pedigree-based approaches. Missing genotypes for the SNPs that passed QC filters were imputed using FImpute [41]. Several commonly used genomic selection models were applied to the data using the R/ BGLR software [42]: rrBLUP, BayesA, BayesB [18] and BayesC [54]. In addition, pedigree-based BLUP [10] was evaluated using the same software. The general form of the fitted models was as in Eq. (1):

$$
\mathbf{l}=\mathbf{X b}+\mathbf{Z} \boldsymbol{\alpha}+\mathbf{e},
$$

where $\mathbf{Z}$ is the incidence matrix relating the underlying liability with the genotypes and $\alpha$ the vector of SNP effects using the corresponding prior distribution for each of the above Bayesian models. The parameters of each model were estimated by MCMC using Gibbs sampling (1.1 million iterations; burn-in: 100,000; thin: 100). Convergence of the resulting posterior distributions was assessed both visually (inspecting the resulting MCMC plots) and analytically using the R/coda v0.19-1 software [44].

Six-fold cross-validation was performed in order to test prediction accuracy of correctly classifying animals in the validation set as resistant or susceptible. The dataset was randomly split into sequential training $(n=1090$ individuals) and validation sets $(\mathrm{n}=218)$. The number of survivors and mortalities in each validation set was proportional to the overall survival of the challenged population. In the validation sets, the phenotypes of the animals were masked, and their (genomic) estimated breeding values-(G)EBV-were estimated based on the prediction model derived from the training set. This cross-validation procedure was repeated five times. Receiver operator characteristic (ROC) curves were used to assess the efficacy of classifying the animals as survivors or mortalities, using either the pedigree- or the genomic-based models. The area under the curve (AUC) metric $[55,56]$ was used 
to interpret the performance of the genomic prediction models, with values of 1 representing the perfect classifier.

\section{Results}

\section{Disease challenge}

The challenge study was conducted for 7 weeks, with mortalities recorded daily. Characteristic clinical signs of $\mathrm{VNN}$ were observed in each disease-challenged tank. In this experiment, the total percentage of mortality reached $48 \%$ (ranging from 40 to $56 \%$ depending on the tank), with four successive peaks of mortality (days 8, 15, 20 and 27); (see Additional file 1: Table S1). Presence of the virus was confirmed in the anterior kidneys and spleens of dead individuals at 1 week post-infection, using primary cell culture coupled with an indirect fluorescent antibody test (IFAT). No mortality was observed in the control tank. Two of the challenge tanks experienced a drop in oxygen concentration at day 27 postinfection, but the fast return to a normal situation within 24 to $48 \mathrm{~h}$ after the incident without observing abnormal behavior or excess mortality led to the conclusion that this temporary oxygen drop did not affect the rest of the experiment. Furthermore, oxygen-related mortalities (day 27 post-infection) were excluded, resulting in 1538 VNN-challenged animals used for downstream analysis.

\section{SNP identification and genotyping}

In total, 6.34 million (s.d. $=3.95$ million) and 2.83 million (s.d. $=1.38$ million) sequence reads passed the QC filters for the parental and offspring samples, respectively. The mean number of putative RAD loci identified was 45,631 (s.d. $=7099)$ and 43,538 (s.d.=4543) for parents and offspring, with mean coverages of $68 \times($ s.d. $=32)$ and $26 \times$ (s.d.=12), respectively. The RAD loci were distributed relatively evenly across the sea bass genome assembly (see Additional file 2: Table S2). Only animals with fewer than $30 \%$ missing genotype data were retained for downstream analysis (67 parents and 1322 offspring). A total of 17,004 putative SNPs were identified, of which 9195 passed the QC filters and were retained for downstream analysis (Table 1) and (see Additional file 3: Table S3). Animals $(\mathrm{n}=11)$ with a genotypic similarity greater than $90 \%$ were discarded as potential replicated samples.

\section{Parentage assignment}

Progeny were assigned to unique parental pairs, allowing for a maximum genotypic error of $3.5 \%$. One thousand two hundred and ninety-seven offspring were uniquely assigned, which revealed that the challenged population (produced from the factorial batch-spawning design described above) comprised 140 full-sib families (48 sires
Table 1 Number of QC-filtered SNPs per chromosome

\begin{tabular}{|c|c|c|}
\hline Chromosome & $\begin{array}{l}\text { Corresponding scaffold } \\
\text { (seabass_v1.0) }\end{array}$ & $\begin{array}{l}\text { Number } \\
\text { of markers }\end{array}$ \\
\hline 1 & HG916827.1 & 330 \\
\hline 2 & HG916828.1 & 393 \\
\hline 3 & HG916829.1 & 321 \\
\hline 4 & HG916830.1 & 387 \\
\hline 5 & HG916831.1 & 367 \\
\hline 6 & HG916832.1 & 355 \\
\hline 7 & HG916833.1 & 293 \\
\hline 8 & HG916834.1 & 375 \\
\hline 9 & HG916835.1 & 237 \\
\hline 10 & HG916836.1 & 340 \\
\hline 11 & HG916837.1 & 370 \\
\hline 12 & HG916838.1 & 268 \\
\hline 13 & HG916839.1 & 371 \\
\hline 14 & HG916840.1 & 367 \\
\hline 15 & HG916841.1 & 336 \\
\hline 16 & HG916842.1 & 254 \\
\hline 17 & HG916843.1 & 145 \\
\hline 18 & HG916844.1 & 420 \\
\hline 19 & HG916845.1 & 390 \\
\hline 20 & HG916846.1 & 404 \\
\hline 21 & HG916847.1 & 376 \\
\hline 22 & HG916848.1 & 334 \\
\hline 23 & HG916849.1 & 322 \\
\hline 24 & HG916850.1 & 259 \\
\hline 25 & HG916851.1 & 1181 \\
\hline Total & & 9195 \\
\hline
\end{tabular}

and 17 dams), with 1 to 32 animals per family and a mean size of 9 (s.d.=8). The contribution of individual dams to the population ranged from 1 to 267 animals, with a mean of 77 (s.d. $=68$ ), while the contribution of sires ranged from 1 to 66 , with a mean of 27 (s.d.=18). Further testing of parentage assignment results using FImpute identified four animals as potentially mis-assigned. These individuals were discarded, which left 1293 animals originating from 48 sires and 17 dams in the final dataset used for the subsequent genetic analyses. The overall survival of these individuals in the VNN challenge was $42 \%$ (Fig. 1). The survival rate varied substantially among the offspring of individual sires (0-100\%) and dams (0-69\%), which indicates genetic variation for resistance to VNN.

\section{Heritability estimates}

Estimates of heritability of overall survival on the underlying liability scale were significant and moderate, at 0.27 (highest posterior density, HPD 95\% interval 0.14-0.40) using the pedigree relationship matrix and at 0.43 (HPD 


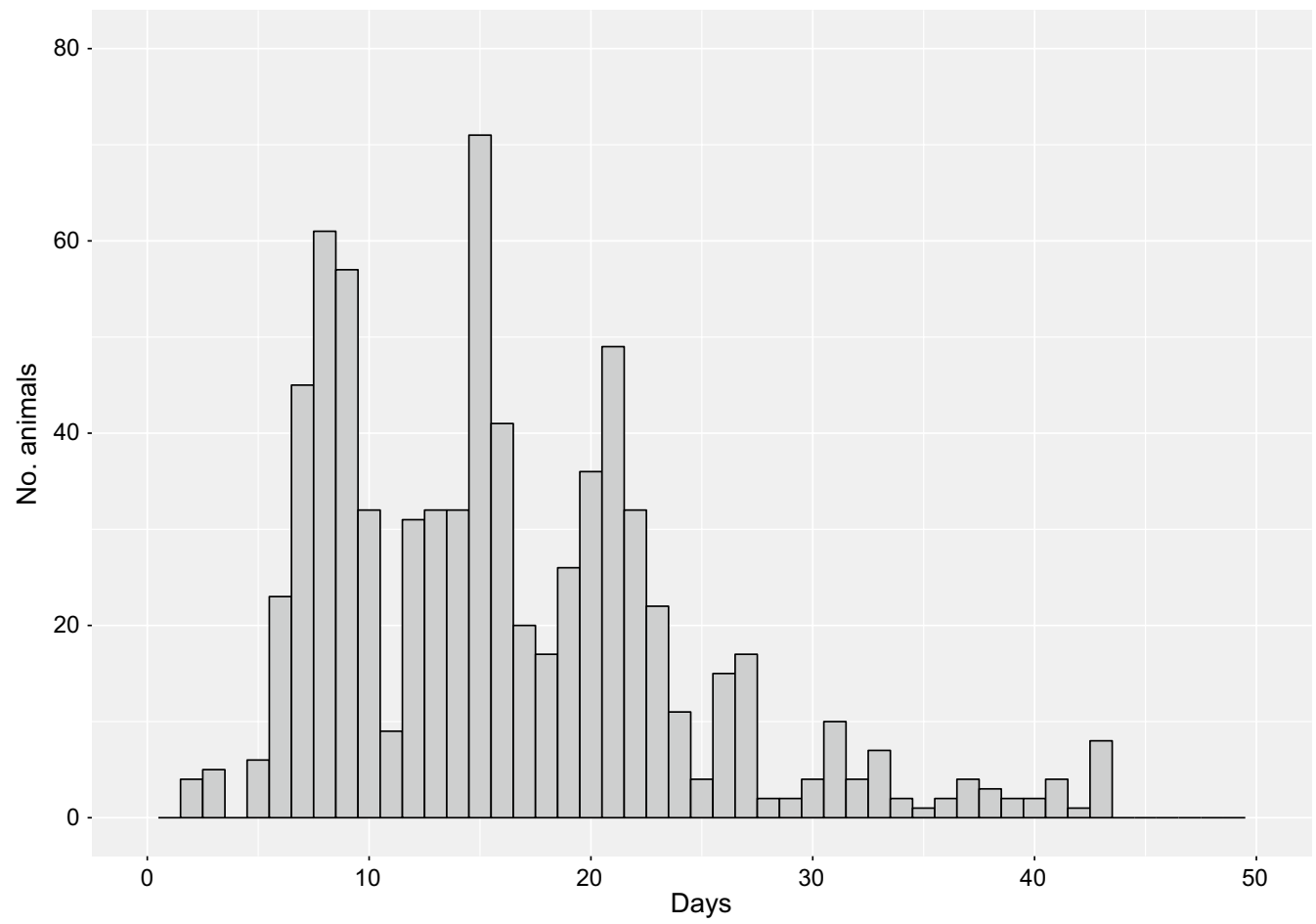

Fig. 1 Daily mortality rates during the VNN disease challenge

95\% interval: 0.29-0.57) using the genomic relationship matrix.

\section{Genome-wide association study (GWAS)}

In the CGWAS, SNPs that exceeded the genome-wide significance level were located on chromosomes 3, 20 and 25 (chromosome 25 corresponds to unassigned scaffolds and contigs of the reference genome assembly; $P<1 \mathrm{e}-05$; $\mathrm{R}^{2}$ : 0.03-0.05; Fig. 2). The WGBLUP analysis provided support for the putative QTL on chromosome 3, with the 0.5-Mb window containing the QTL-associated SNPs estimated to explain approximately $4 \%$ of the genetic variation. The putative QTL on chromosomes 20 and 25 were estimated to explain 1.5 and $2 \%$ of the genetic variation, respectively, in the WGLUP. Additional putative QTL regions that explained more than $2 \%$ of the additive genetic variance were identified on chromosomes 9,18 , 21 and 22 (Fig. 3).

\section{Genomic prediction}

The genomic prediction models successfully classified animals of the validation set with a success rate ranging from 67 to $70 \%$, representing improvements of 8 to $13 \%$ over pedigree-based BLUP (PBLUP) (Table 2, Fig. 4). The best classifications were obtained with the Bayes $\mathrm{A}$ and Bayes B methods, corresponding to a success rate of $70 \%$
(Table 2) for correct classification (based on the AUC values of the ROC curves; Fig. 4).

\section{Discussion}

European sea bass is a farmed species of paramount importance for Mediterranean aquaculture. Breeding for improved genetic resistance to $\mathrm{VNN}$ offers a promising avenue for reducing economic losses due to this disease and for improving animal welfare. A small number of family-based selective breeding programs exist for sea bass, with growth and disease resistance as the major target traits. Applying genomic information to selective breeding schemes facilitates direct selection for favorable alleles at major QTL (marker-assisted selection) and/or incorporation of genome-wide markers into the prediction of breeding values (genomic selection). Genomic prediction methods have been repeatedly shown to increase selection accuracy compared to pedigree-based approaches in aquaculture [29, $30,57]$, which should translate to faster rates of genetic gain. High-density SNP arrays have been used for genomic selection, but these require substantial prior investment for development and application. As such, SNP arrays may be too expensive for routine genotyping in small to medium scale selective breeding programs, and more cost-effective genotyping methods are highly desirable. Techniques such as RAD sequencing, which 


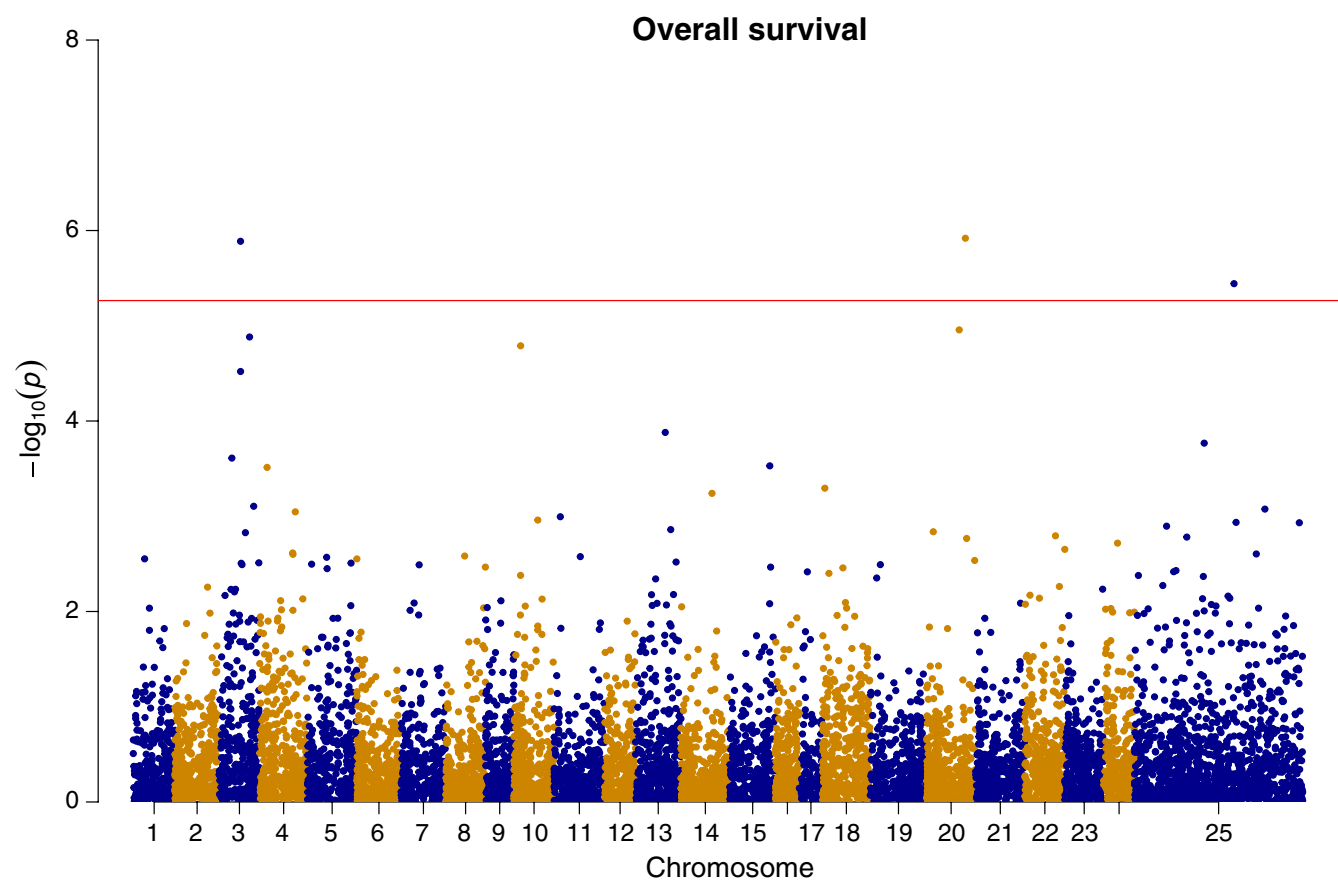

Fig. 2 Genome-wide association plot for survival during the VNN challenge using single SNP GWAS

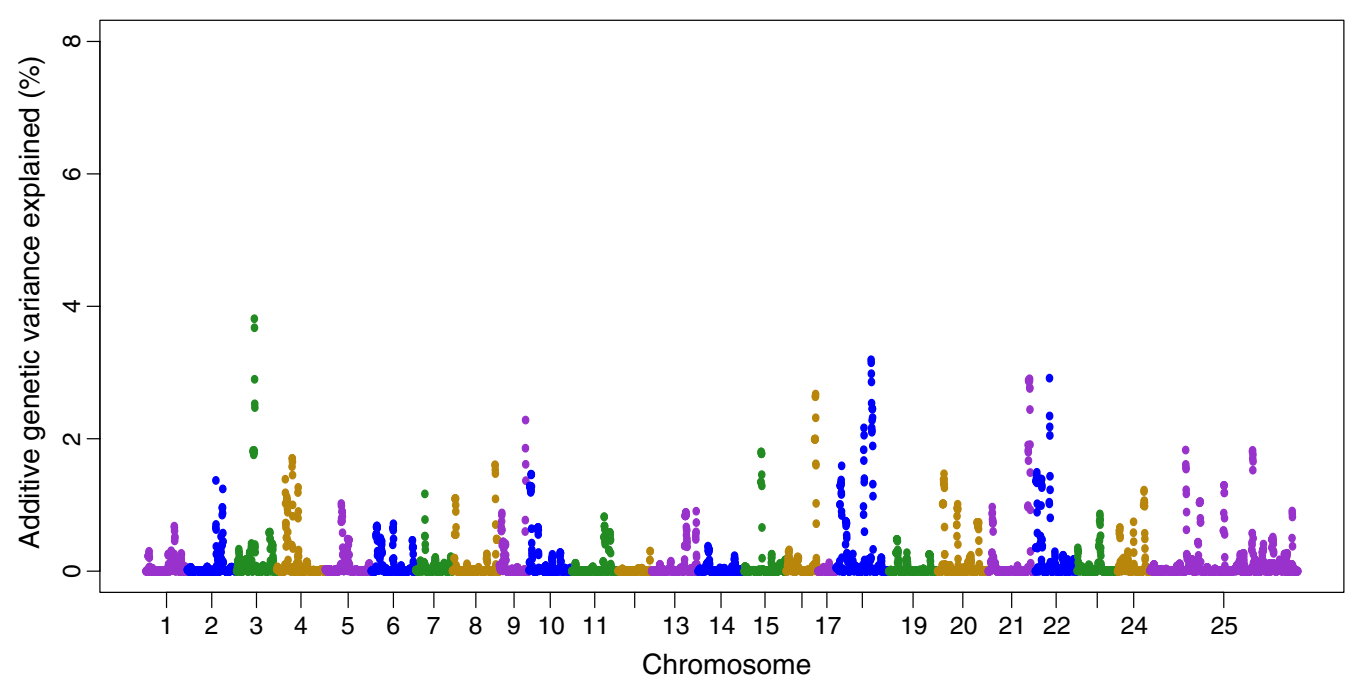

Fig. 3 Genome-wide association plot for WGBLUP for survival during the VNN challenge, with the explained additive genetic variance calculated using non-overlapping windows of $0.5 \mathrm{Mb}$

require minimal prior investment, offer a cost effective alternative of generating genome-wide SNP datasets, even in the absence of prior genomic resources [22]. $\mathrm{RAD}$ sequencing and other genotyping-by-sequencing approaches are therefore becoming increasingly commonplace in aquaculture genetics and breeding [23].
Our study is the first application of high throughput sequencing to study genetic resistance of sea bass to VNN. The estimated heritability of resistance was moderate to high (ranging from 0.27 to 0.43 ). The heritability estimate obtained by using the pedigree relationship matrix (0.27) was in accordance with a recently 
Table 2 Percentage of VNN challenged sea bass with correctly predicted survival status for pedigreebased (PBLUP) and genomic prediction methods

\begin{tabular}{llllll}
\hline Replication & PBLUP & rrBLUP & Bayes A & Bayes B & Bayes C \\
\hline $1^{\text {st }}$ & 0.63 & 0.67 & 0.70 & 0.71 & 0.67 \\
$2^{\text {nd }}$ & 0.62 & 0.66 & 0.69 & 0.70 & 0.67 \\
$3^{\text {rd }}$ & 0.62 & 0.67 & 0.70 & 0.69 & 0.68 \\
$4^{\text {th }}$ & 0.62 & 0.66 & 0.70 & 0.70 & 0.68 \\
$5^{\text {th }}$ & 0.62 & 0.67 & 0.70 & 0.70 & 0.69 \\
Mean & 0.62 & 0.67 & 0.70 & 0.70 & 0.68 \\
\hline
\end{tabular}

Values obtained using area under curve (AUC) from ROC curves

Five replicates of sixfold cross-validation

estimated heritability $(0.26)$ for resistance to $\mathrm{VNN}$ in several sea bass populations, also using pedigree [8]. These significant heritability estimates indicate that the aquaculture industry can benefit from the application of selective breeding for sea bass resistant to VNN. The reason that a higher heritability estimate was obtained when using the genomic relationship matrix (0.43) is not clear, but it is plausible that linkage disequilibrium generated by recent selective breeding may cause overestimation of additive genetic variance when using a genomic relationship matrix [58].

The CGWAS identified genome-wide significant QTL in several genomic regions (chromosomes $3,20,25$, with chromosome 25 representing unassigned genome contigs and scaffolds). The single SNP CGWAS approach may lack statistical power to detect QTL when compared to methods in which all SNPs are used simultaneously, because of a false assumption of independence between SNPs [49]. WGBLUP is an approach that combines the computational efficiency of GBLUP with an increase in statistical power for QTL detection [50].

The genomic region that explained the highest percentage of additive genetic variance in the WGBLUP $(\sim 4 \%)$ was located on chromosome 3 and was also detected in the CGWAS. However, this may be an overestimation of the explained additive variance because threshold models often lead to overestimates of genetic variance [59]. In spite of some degree of discrepancy between the two GWAS approaches used, both highlight significant QTL that may play a role in host resistance to $\mathrm{VNN}$, but also suggest that the trait is polygenic or 'oligogenic' in nature. In a similar study on resistance to VNN in Asian sea bass, no major QTL were identified [60], which supports the hypothesis that genetic resistance to VNN may be under

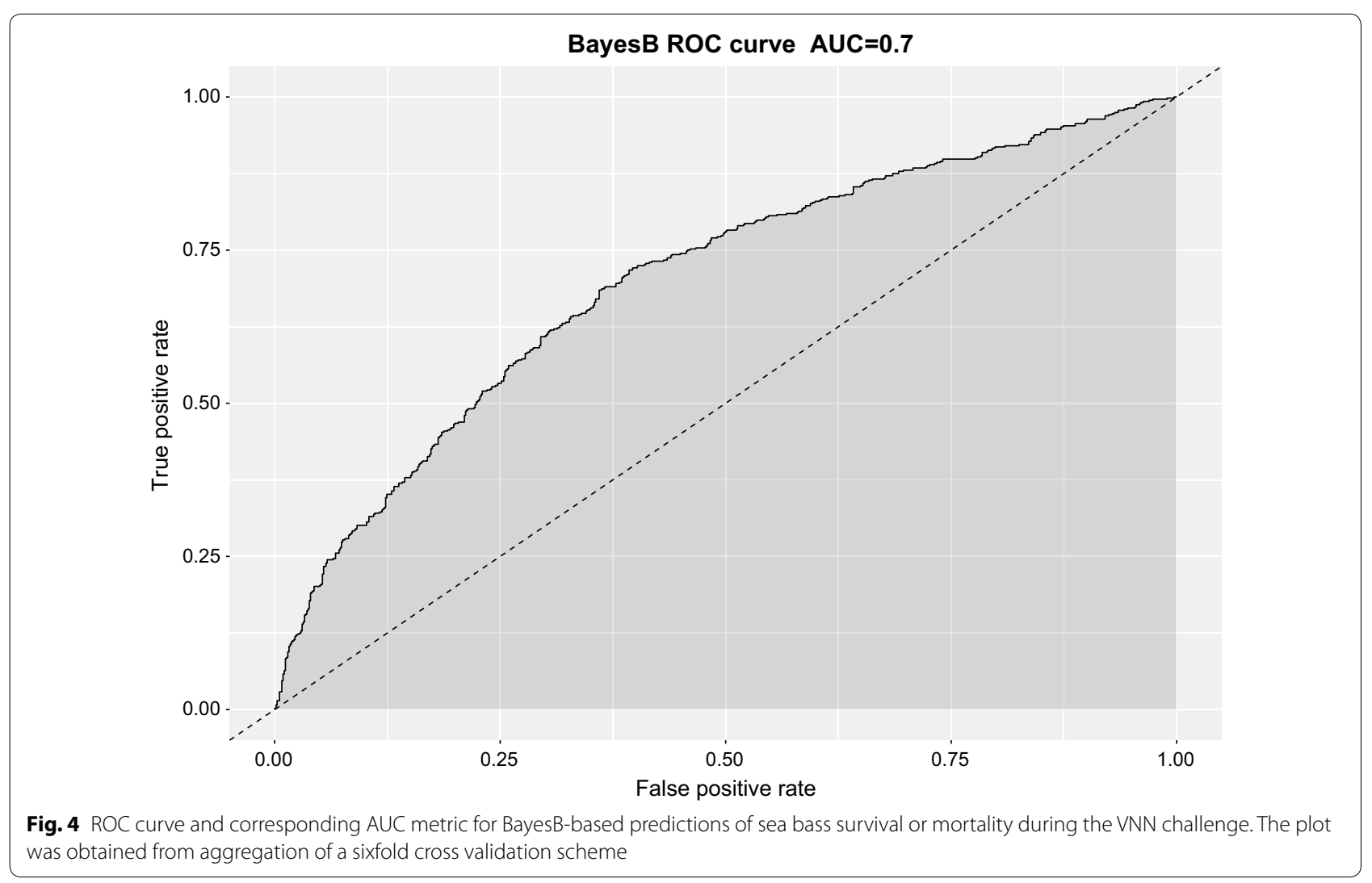


the control of many genomic regions, each with a minor to moderate effect. In such situations, genomic selection is likely the most appropriate approach for using genetic markers to improve selective breeding.

The results from the genomic prediction approach used in the current study were encouraging for practical implementation of genomic selection for genetic resistance in sea bass. For traits like disease resistance, which cannot be measured directly on selection candidates, genomic selection benefits from the use of within-family genetic variation, compared to pedigree-based BLUP, which uses between-family genetic variation only [61]. Improvements in selection accuracies due to genomic prediction for disease resistance traits have been documented in various finfish aquaculture species, including Atlantic salmon [21], rainbow trout [29], and gilthead sea bream [30]. The AUC metrics derived from the ROC curves take the rate of both false positives and false negatives into consideration and have been routinely used to test the efficacy of prediction models for binary traits both in humans [56] and in livestock [62]. Interestingly, our study resulted in higher AUC values compared to livestock studies that used similar sample sizes and SNP density $[62,63]$, with the best performing models on average correctly classifying $70 \%$ of the validation set. This may be related to long-range linkage disequilibrium as a result of close genetic relationships between training and validation sets, which include large numbers of full and half siblings. Genetic relationships between reference and test populations are known to be a key factor that influences prediction accuracies in genomic selection [64]. However, reliance on these close genetic relationships for genomic prediction implies that generalization of these results to other populations and reference population designs should be undertaken with caution. Furthermore, additional testing of genomic prediction at varying SNP densities on a wider population is required to ascertain the appropriate SNP density for commercial application of genomic selection.

\section{Conclusions}

We applied RAD sequencing to study the genetic basis of resistance to VNN in a large sample of juvenile sea bass derived from a commercial breeding program. RADderived SNPs allowed us to perform pedigree assignment in this batch-spawning species, estimate genetic parameters, perform GWAS, and test genomic selection. Genome-wide significant QTL were identified with minor to moderate effects on host resistance, on chromosomes 3, 20 and on an unassigned genome scaffolds. Genomic prediction using the RAD genotype data was effective, demonstrating significant improvement in prediction accuracy, and facilitating classification of surviving and mortality fish with a success rate of $70 \%$ using the cross-validation approach. This highlights the utility of genotyping-by-sequencing for genomic prediction of disease resistance in aquaculture species, and demonstrates that genomic selection can be an effective method for improving resistance to one of the most problematic infectious diseases in sea bass aquaculture.

\section{Additional files}

Additional file 1: Table S1. VNN challenged sea bass. Phenotypic information of VNN challenged sea bass and pedigree structure.

Additional file 2: Table S2. Identified SNPs. Sequence information of identified SNPS.

Additional file 3: Table S3. SNP location. Location of SNPs on the sea bass reference genome $\mathbf{v} 1 \_$.

\section{Authors' contributions}

$\mathrm{SC}, \mathrm{AB}, \mathrm{JB}, \mathrm{PH}, \mathrm{RH}$ conceived the study, contributed to the design of the experimental structure. TM and JC carried out the challenge experiment. CP carried out DNA extractions, RAD library preparation, and sequence data processing. FA and MV carried out the parentage assignment. CP and $\mathrm{RH}$ carried out the quantitative genetic analyses. All authors contributed to draft the manuscript. All authors read and approved the final manuscript.

\section{Author details}

${ }^{1}$ The Roslin Institute, Royal (Dick) School of Veterinary Studies, University of Edinburgh, Easter Bush, Midlothian EH25 9RG, Scotland, UK. ${ }^{2}$ Ferme Marine De Douhet, BP 4, 17840 La Brée Les Bains, France. ${ }^{3}$ SYSAAF, LPGP-INRA, Campus de Beaulieu, 35042 Rennes Cedex, France. ${ }^{4}$ French Agency for Food, Environmental and Occupational Health and Safety (ANSES), Ploufragan-Plouzané Laboratory, Viral Fish Pathology Unit, National Reference Laboratory for Regulated Fish Diseases, Bretagne Loire University, Technopôle BrestIroise, BP 70, 29280 Plouzané, France. ${ }^{5}$ MARBEC, Université de Montpellier, Ifremer-CNRS-IRD-UM, Palavas-les-Flots, France. ${ }^{6} \mathrm{GABI}$, INRA, AgroParisTech, Université Paris-Saclay, 78350 Jouy-en-Josas, France.

\section{Acknowledgements}

The authors are supported by funding from the European Union's Seventh Framework Programme (FP7 2007-2013) under Grant Agreement No. 613611 (FISHBOOST). RH and CP are supported by Biotechnology and Biological Sciences Research Council Institute StrategicProgramme Grants (BBS/ E/D/20002172 and BBS/E/D/30002275)

Competing interests

The authors declare that they have no competing interests.

\section{Ethics approval and consent to participate}

All experiments on animals were carried out in strict accordance with the European guidelines and recommendations on animal experimentation and welfare and animal experiment procedures were approved by the ethics committees on animal experimentation ANSES/ENVA/UPC no. 16 and were authorized by the "Ministère de l'Education Nationale, de l'Enseignement Supérieur et de la Recherche", undernumber 29/01/13-5.

\section{Publisher's Note}

Springer Nature remains neutral with regard to jurisdictional claims in published maps and institutional affiliations.

Received: 9 October 2017 Accepted: 31 May 2018

Published online: 08 June 2018 


\section{References}

1. FEAP_Production data. http://www.feap.info/default.asp?SHORT $\mathrm{CUT}=582$. Accessed 28 May 2018.

2. Doan QK, Vandeputte M, Chatain B, Morin T, Allal F. Viral encephalopathy and retinopathy in aquaculture: a review. J Fish Dis. 2017:40:717-42.

3. Le Breton A, Grisez L, Sweetman J, Ollevier F. Viral nervous necrosis (VNN) associated with mass mortalities in cage-reared sea bass, Dicentrarchus labrax (L.). J Fish Dis. 1997;20:145-51.

4. Vendramin N, Zrncic S, Padrós F, Oraic D, Le Breton A, Zarza C, et al. Fish health in Mediterranean aquaculture, past mistakes and future challenges. Bull Eur Assoc Fish Pathol. 2016;36:38-45.

5. Brudeseth BE, Wiulsrod R, Fredriksen BN, Lindmo K, Lokling KE, Bordevik $M$, et al. Status and future perspectives of vaccines for industrialised finfish farming. Fish Shellfish Immunol. 2013;35:1759-68.

6. Bishop SC, Woolliams JA. Genomics and disease resistance studies in livestock. Livest Sci. 2014;166:190-8.

7. Ødegård J, Baranski M, Gjerde B, Gjedrem T. Methodology for genetic evaluation of disease resistance in aquaculture species: challenges and future prospects. Aquacult Res. 2011;42:103-14.

8. Doan QK, Vandeputte M, Chatain B, Haffray P, Vergnet A, Breuil G, et al Genetic variation of resistance to Viral Nervous Necrosis and genetic correlations with production traits in wild populations of the European sea bass (Dicentrarchus labrax). Aquaculture. 2017;478:1-8.

9. Davey JW, Hohenlohe PA, Etter PD, Boone JQ, Catchen JM, Blaxter ML. Genome-wide genetic marker discovery and genotyping using nextgeneration sequencing. Nat Rev Genet. 2011;12:499-510.

10. Henderson CR. Best linear unbiased estimation and prediction under a selection model. Biometrics. 1975;31:423-47.

11. Goddard ME, Hayes BJ. Mapping genes for complex traits in domestic animals and their use in breeding programmes. Nat Rev Genet. 2009:10:381-91.

12. de Los Campos G, Hickey JM, Pong-Wong R, Daetwyler HD, Calus MPL. Whole-genome regression and prediction methods applied to plant and animal breeding. Genetics. 2013;193:327-45.

13. Habier D, Fernando RL, Garrick DJ. Genomic BLUP decoded: a look into the black box of genomic prediction. Genetics. 2013;194:597-607.

14. Houston RD, Haley CS, Hamilton A, Guy DR, Tinch AE, Taggart JB, et al. Major quantitative trait loci affect resistance to infectious pancreatic necrosis in Atlantic salmon (Salmo salar). Genetics. 2008;178:1109-15.

15. Moen T, Baranski M, Sonesson AK, Kjøglum S. Confirmation and finemapping of a major QTL for resistance to infectious pancreatic necrosis in Atlantic salmon (Salmo salar): population-level associations between markers and trait. BMC Genomics. 2009;10:368.

16. Palaiokostas C, Bekaert M, Davie A, Cowan ME, Oral M, Taggart JB, et al. Mapping the sex determination locus in the Atlantic halibut (Hippoglossus hippoglossus) using RAD sequencing. BMC Genomics. 2013;14:566.

17. Gonen S, Baranski M, Thorland I, Norris A, Grove H, Arnesen P, et al. Mapping and validation of a major QTL affecting resistance to pancreas disease (salmonid alphavirus) in Atlantic salmon (Salmo salar). Heredity (Edinb). 2015;115:405-14.

18. Meuwissen THE, Hayes BJ, Goddard ME. Prediction of total genetic value using genome-wide dense marker maps. Genetics. 2001;157:1819-29.

19. Ødegård J, Moen T, Santi N, Korsvoll SA, Kjøglum S, Meuwissen THE. Genomic prediction in an admixed population of Atlantic salmon (Salmo salar). Front Genet. 2014:5:402.

20. Tsai HY, Hamilton A, Tinch AE, Guy DR, Gharbi K, Stear MJ, et al. Genome wide association and genomic prediction for growth traits in juvenile farmed Atlantic salmon using a high density SNP array. BMC Genomics. 2015;16:969.

21. Tsai HY, Hamilton A, Tinch AE, Guy DR, Bron JE, Taggart JB, et al. Genomic prediction of host resistance to sea lice in farmed Atlantic salmon populations. Genet Sel Evol. 2016:48:47.

22. Baird NA, Etter PD, Atwood TS, Currey MC, Shiver AL, Lewis ZA, et al. Rapid SNP discovery and genetic mapping using sequenced RAD markers. PLoS One. 2008;3:e3376.

23. Robledo D, Palaiokostas C, Bargelloni L, Martínez P, Houston R. Applications of genotyping by sequencing in aquaculture breeding and genetics. Rev Aquac. 2017. https://doi.org/10.1111/raq.12193.

24. Campbell NR, LaPatra SE, Overturf K, Towner R, Narum SR. Association mapping of disease resistance traits in rainbow trout using restriction site associated DNA sequencing. G3 (Bethesda). 2014;4:2473-81.
25. Palti Y, Vallejo RL, Gao G, Liu S, Hernandez AG, Rexroad CE 3rd, et al. Detection and validation of QTL affecting bacterial cold water disease resistance in Rainbow trout using restriction-site associated DNA sequencing. PLoS One. 2015;10:e0138435.

26. Barria A, Christensen KA, Yoshida GM, Correa K, Jedlicki A, Lhorente JP, et al. Genomic predictions and genome-wide association study of resistance against Piscirickettsia salmonis in Coho Salmon (Oncorhynchus kisutch) using ddRAD sequencing. G3 (Bethesda). 2018;8:1183-94.

27. Dou J, Li X, Fu Q, Jiao W, Li Y, Li T, et al. Evaluation of the 2b-RAD method for genomic selection in scallop breeding. Sci Rep. 2016;6:19244.

28. Vallejo RL, Leeds TD, Fragomeni BO, Gao G, Hernandez AG, Misztal I, et al. Evaluation of genome-enabled selection for bacterial cold water disease resistance using progeny performance data in Rainbow trout: insights on genotyping methods and genomic prediction models. Front Genet. 2016;7:96.

29. Vallejo RL, Leeds TD, Gao G, Parsons JE, Martin KE, Evenhuis JP, et al. Genomic selection models double the accuracy of predicted breeding values for bacterial cold water disease resistance compared to a traditional pedigree-based model in rainbow trout aquaculture. Genet Sel Evol. 2017:49:17.

30. Palaiokostas C, Ferraresso S, Franch R, Houston RD, Bargelloni L. Genomic prediction of resistance to Pasteurellosis in Gilthead Sea Bream (Sparus aurata) using 2b-RAD sequencing. G3 (Bethesda). 2016;6:3693-700.

31. Palaiokostas C, Kocour M, Prchal M, Houston RD. Accuracy of genomic evaluations of juvenile growth rate in common carp (Cyprinus carpio) using genotyping by sequencing. Front Genet. 2018;9:82.

32. Castric J, Thiéry R, Jeffroy J, de Kinkelin P, Raymond JC. Sea bream Sparus aurata, an asymptomatic contagious fish host for nodavirus. Dis Aquat Organ. 2001:47:33-8.

33. Thiéry R, Cozien J, de Boisséson C, Kerbart-Boscher S, Névarez L. Genomic classification of new betanodavirus isolates by phylogenetic analysis of the coat protein gene suggests a low host-fish species specificity. J Gen Virol. 2004;85:3079-87.

34. Etter PD, Bassham S, Hohenlohe PA, Johnson EA, Cresko WA. SNP discovery and genotyping for evolutionary genetics using RAD sequencing. Methods Mol Biol. 2011;772:157-78.

35. Houston RD, Davey JW, Bishop SC, Lowe NR, Mota-Velasco JC, Hamilton A, et al. Characterisation of QTL-linked and genome-wide restriction siteassociated DNA (RAD) markers in farmed Atlantic salmon. BMC Genomics. 2012:13:244.

36. Tine M, Kuhl H, Gagnaire PA, Louro B, Desmarais E, Martins RS, et al. European sea bass genome and its variation provide insights into adaptation to euryhalinity and speciation. Nat Commun. 2014;5:5770.

37. Langmead B, Salzberg SL. Fast gapped-read alignment with Bowtie 2. Nat Methods. 2012;9:357-9.

38. Catchen JM, Amores A, Hohenlohe P, Cresko W, Postlethwait JW. Stacks: building and genotyping loci de novo from short-read sequences. G3 (Bethesda). 2011;1:171-82.

39. Hohenlohe PA, Bassham S, Etter PD, Stiffler N, Johnson EA, Cresko WA Population genomics of parallel adaptation in threespine stickleback using sequenced RAD tags. PLoS Genet. 2010:6:e1000862.

40. Ferdosi MH, Kinghorn BP, van der Werf JHJ, Lee SH, Gondro C. hsphase: an $\mathrm{R}$ package for pedigree reconstruction, detection of recombination events, phasing and imputation of half-sib family groups. BMC Bioinformatics. 2014:15:172

41. Sargolzaei M, Chesnais JP, Schenkel FS. A new approach for efficient genotype imputation using information from relatives. BMC Genomics. 2014;15:478.

42. Pérez P, de Los Campos G. Genome-wide regression and prediction with the BGLR statistical package. Genetics. 2014;198:483-95.

43. VanRaden PM. Efficient methods to compute genomic predictions. J Dairy Sci. 2008:91:4414-23.

44. Plummer M, Best N, Cowles K, Vines K. CODA: convergence diagnosis and output analysis for MCMC. R News. 2006;6:7-11.

45. Goldstein H, Browne W, Rasbash J. Partitioning variation in multilevel models. Underst Stat. 2002:1:223-31.

46. Nakagawa S, Schielzeth H. A general and simple method for obtaining R2 from generalized linear mixed-effects models. Methods Ecol Evol. 2013:4:133-42

47. Perdry H, Dandine-Roulland C, Bandyopadhyay D, Kettner L. Package 'gaston': Genetic data handling (QC, GRM, LD, PCA) and linear mixed models. 
Version 1.5.3. ftp://cran.r-project.org/pub/R/web/packages/gaston/gasto n.pdf.

48. Chen H, Wang C, Conomos MP, Stilp AM, Li Z, Sofer T, et al. Control for population structure and relatedness for binary traits in genetic association studies via logistic mixed models. Am J Hum Genet. 2016;98:653-66.

49. Wang H, Misztal I, Aguilar I, Legarra A, Mui WM. Genome-wide association mapping including phenotypes from relatives without genotypes. Genet Res (Camb). 2012;94:73-83.

50. Zhang X, Lourenco D, Aguilar I, Legarra A, Misztal I. Weighting strategies for single-step genomic BLUP: an iterative approach for accurate calculation of GEBV and GWAS. Front Genet. 2016;7:151.

51. Misztal I, Tsuruta S, Strabel T, Auvray B, Druet T, Lee DH. BLUPF90 and related programs (BGF90). In: Proceedings of the 7 th world congress on genetics applied to livestock production: 19-23 August 2002; Montpellier; 2002.

52. Tsuruta S, Misztal I. THRGIBBS1F90 for estimation of variance components with threshold and linear models. In Proceedings of the 8th world congress on genetics applied to livestock production: 13-18 August, 2006; Belo Horizonte; 2006.

53. Aguilar I, Misztal I, Legarra A, Tsuruta S. Efficient computation of the genomic relationship matrix and other matrices used in single-step evaluation. J Anim Breed Genet. 2011;128:422-8.

54. Habier D, Fernando RL, Kizilkaya K, Garrick DJ. Extension of the bayesian alphabet for genomic selection. BMC Bioinformatics. 2011;12:186.

55. Hanley AJ, McNeil JB. The meaning and use of the area under a receiver operating characteristic (ROC) curve. Radiology. 1982;143:29-36.

56. Wray NR, Yang J, Goddard ME, Visscher PM, Kimberly R. The genetic interpretation of area under the ROC curve in genomic profiling. PLoS Genet. 2010;6:e1000864.
57. Correa K, Bangera R, Figueroa R, Lhorente JP, Yanez JM. The use of genomic information increases the accuracy of breeding value predictions for sea louse (Caligus rogercresseyi) resistance in Atlantic salmon (Salmo salar). Genet Sel Evol. 2017:49:15.

58. Fernando RL, Cheng H, Sun X, Garrick DJ. A comparison of identityby-descent and identity-by-state matrices that are used for genetic evaluation and estimation of variance components. J Anim Breed Genet. 2017;134:213-23.

59. Ødegård J, Meuwissen THE, Heringstad B, Madsen P. A simple algorithm to estimate genetic variance in an animal threshold model using Bayesian inference. Genet Sel Evol. 2010;42:29.

60. Wang L, Liu P, Huang S, Ye B, Chua E, Wan ZY, et al. Genome-wide association study identifies loci associated with resistance to viral nervous necrosis disease in Asian Seabass. Mar Biotechnol (NY). 2017;19:255-65.

61. Lillehammer M, Meuwissen THE, Sonesson AK. A low-marker density implementation of genomic selection in aquaculture using within-family genomic breeding values. Genet Sel Evol. 2013;45:39.

62. Tsairidou S, Woolliams JA, Allen AR, Skuce RA, McBride SH, Wright DM et al. Genomic prediction for tuberculosis resistance in dairy cattle. PLoS One. 2014;9:e96728.

63. Zare Y, Shook GE, Collins MT, Kirkpatrick BW. Genome-wide association analysis and genomic prediction of Mycobacterium avium subspecies paratuberculosis infection in US Jersey cattle. PLoS One. 2014;9:e88380.

64. Meuwissen TH, Hayes BJ, Goddard ME. Accelerating improvement of livestock with genomic selection. Annu Rev Anim Biosci. 2013;1:221-37.
Ready to submit your research? Choose BMC and benefit from:

- fast, convenient online submission

- thorough peer review by experienced researchers in your field

- rapid publication on acceptance

- support for research data, including large and complex data types

- gold Open Access which fosters wider collaboration and increased citations

- maximum visibility for your research: over 100M website views per year

At BMC, research is always in progress.

Learn more biomedcentral.com/submissions 